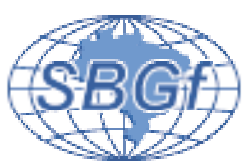

\title{
REFLEXÃO SÍSMICA RASA ACIMA DO ÂNGULO CRÍTICO DE INCIDÊNCIA: AQUISIÇÃO PROCESSAMENTO E INTERPRETAÇÃO
}

Liliana Alcazar Diogo, IAG/USP- Departamento de Geofísica

Copyright 2004, SBGf - Sociedade Brasileira de Geofísica

Este texto foi preparado para a apresentação no I Simpósio Regional da Sociedade Brasileira de Geofísica, São Paulo, 26-28 de setembro de 2004. Seu conteúdo fol revisado pela Comissão Tecno-científica do I SR-SBGf mas não necessariamente representa a opinião da SBGf ou de seus associados. E proibida a reprodução total ou parcial deste material para propósitos comerciais sem prévia autorização da SBGf.

\section{Resumo}

Neste trabalho discute-se sobre a viabilidade, vantagens e inconvenientes em se adquirir, processar e interpretar registros sísmicos de reflexões provenientes de alvos rasos detectados em afastamentos fonte-receptor longos, maiores do que a distância crítica de refração.

São duas as motivações para o desenvolvimento deste estudo: i) possibilitar o uso do método sísmico de reflexão em terrenos que não apresentam uma resposta sísmica com conteúdo de freqüência elevado o suficiente para se observar as reflexões nos afastamentos curtos, onde a energia do groundroll, em geral, predomina, e ii) explorar as mudanças na fase do pulso refletido para ângulos de incidência maiores que o ângulo crítico para a estimativa da velocidade da onda de cisalhamento e da densidade do meio, assumindo que a velocidade da onda $P$ é conhecida. Desta forma, seria possível a obtenção dos módulos de elasticidade, importantes para a caracterização e avaliação das propriedades mecânicas de maciços, para aplicações em geotecnia.

Utilizando-se dados sintéticos de reflexão, o processo de análise de velocidades e a imagem resultante nas seções empilhadas em tempo foram comparados para simulações dos levantamentos de reflexão executados abaixo e acima do ângulo crítico de incidência $\left(\theta_{c}\right)$. Observou-se que a imagem do refletor para os sinais abaixo e acima de $\theta_{c}$ podem ser equivalentes. Entretanto, não é possível obter o valor correto da velocidade de propagação da onda, através dos procedimentos convencionais, para as reflexões acima de $\theta c$. Desta forma, para a conversão tempo-profundidade da seção acima de $\theta_{c}$ é necessário obter as velocidades da onda $P$ através de outro método.

As investigações para a estimativa da densidade e dos módulos de elasticidade do meio explorando a defasagem do pulso refletido acima do ângulo crítico ainda estão em andamento. $\mathrm{O}$ estudo realizado até o momento apresenta resultados promissores nesse sentido.

\section{Introdução}

Os ruídos coerentes presentes nos registros sísmicos em afastamentos próximos da fonte, principalmente devidos ao groundroll, onda direta e onda área, mascaram a energia refletida, sendo que em muitos casos nenhum processamento digital é suficientemente efetivo para realçar as reflexões. Esta é uma situação comum em levantamentos de reflexão sísmica rasa, especialmente em terrenos que não apresentam uma resposta sísmica com conteúdo de freqüência elevado o suficiente para se observar as reflexões nos afastamentos curtos, onde a energia do groundroll, em geral, predomina.

Em alguns casos as reflexões apresentam-se em afastamentos longos com maior nitidez, aparentando maior amplitude, por estar livre da superposição de qualquer ruído coerente. Entretanto o caráter do pulso refletido e a curva de tempo de percurso nesses afastamentos sofrem modificações em relação às reflexões que chegam nos afastamentos curtos.

Hunter et al. (1980) comentam sobre as mudanças de amplitude e fase do sinal refletido com a variação do afastamento fonte-receptor e sugerem que o arranjo sísmico deve ser posicionado dentro de um intervalo de afastamentos em que o efeito dessas variações for menor. Pullan \& Hunter (1985) examinaram através de sismogramas sintéticos o efeito da partição de energia sobre o caráter do pulso refletido para modelos realísticos na escala de investigação rasa.

Neste trabalho, são discutidos alguns aspectos práticos para a aquisição do sinal refletido acima do ângulo crítico e investigadas as conseqüências no processamento e na interpretação das reflexões acima do ângulo crítico.

Um dos principais inconvenientes em se trabalhar com afastamentos longos é a superestimativa dos valores da velocidade através dos procedimentos convencionais que se baseiam na equação da hipérbole para a curva de tempo de percurso. Além disso a mudança na forma do pulso refletido para ângulos acima do ângulo crítico também contribui para a determinação incorreta dos valores das velocidades de RMS (root mean square). Por outro lado, a imagem observada na seção empilhada em tempo pode ser representativa das estruturas em subsuperfície, independente da velocidade de empiIhamento. O problema em questão é a conversão da seção em tempo para profundidade, o que requer a determinação da velocidade da onda $P$ através de outras observações, por exemplo, da refração no topo da camada acima do refletor em questão.

A partição de energia na interface, quantificada pelo coeficiente de reflexão é o principal fator responsável pelas mudanças no caráter do pulso refletido para ângulos de incidência maiores do que o ângulo crítico $\left(\theta_{c}\right)$. O coeficiente de reflexão depende do ângulo de incidência, das densidades $(\rho)$ dos meios e das velocidades das ondas $\mathrm{P}$ e $\mathrm{S}$ ( $\mathrm{Vp}$ e $\mathrm{Vs}$ ) acima e abaixo do refletor. Assumindo que a Vp é conhecida, está sendo investigada a possibilidade em se estimar Vs e $\rho$, comparando-se a forma do pulso modelado com o pulso real. 


\section{Procedimentos de Aquisição}

A aquisição de sismogramas para análise de ruído (walkaway noise test) costuma ser um procedimento rotineiro para o planejamento de um levantamento de reflexão sísmica rasa. O principal objetivo é a determinação da janela de afastamentos ideais para se observar as reflexões livres da superposição dos ruídos coerentes. Como neste trabalho se está avaliando a possibilidade em se adquirir o sinal refletido acima do ângulo crítico, a janela ideal neste caso refere-se ao intervalo de afastamentos, dentro do qual, o pulso refletido não apresente variações bruscas de fase.

O levantamento de reflexão será simulado segundo a técnica CMP, cujo objetivo do procedimento de aquisição é amostrar várias vezes o mesmo ponto em subsuperfície, para diferentes afastamentos fontereceptor. O procedimento convencional para a aquisição CMP, consolidado para levantamentos que visam à exploração de petróleo, consiste em executar, continuamente ao longo da linha, uma seqüência de pontos de tiro, sendo que todo o arranjo de receptores registrados é deslocado do mesmo intervalo entre os pontos de tiro.

A execução do procedimento acima, do ponto de vista prático, considerando o tempo de aquisição no campo, requer um sismógrafo com um número de canais pelo menos quatro vezes maior que a multiplicidade desejada. Do contrário, suponha que está sendo utilizado um sismógrafo de 24 canais e se deseja uma cobertura de $1200 \%$ para os pontos amostrados em subsuperfície. Com essa configuração, a cada ponto de tiro, deve-se mover fisicamente um geofone para o final da linha, desconectando e reconectando o cabo a todos os geofones.

Um procedimento alternativo para a aquisição CMP foi empregado por Le Diagon (2000), com o objetivo de reduzir o tempo de execução do levantamento de campo, em função do número limitado de canais de registro do sismógrafo. Para se obter uma cobertura de $1200 \%$ com 24 canais disponíveis, o procedimento consiste em manter fixo o arranjo de geofones e mover o ponto de tiro na direção do arranjo. Depois de executados 12 pontos de tiro (o que equivale à metade do número de canais registrados) espaçados com o mesmo intervalo entre os receptores, movem-se os primeiros 12 geofones para o final do arranjo, e prossegue-se com a execução de novos 12 pontos de tiro. Esse procedimento difere da técnica convencional por não manter o mesmo afastamento mínimo em todos os pontos de tiro do levantamento. Como o conjunto de geofones é mantido na mesma posição para uma seqüência de pontos de tiro, denominou-se nesse trabalho cada arranjo fixo de geofones por "base" e o procedimento de aquisição por levantamento CMP "base-fixa".

\section{Processamento e Interpretação}

Utilizando-se dados sintéticos de reflexão, avaliou-se a utilização dos processos convencionais para o processamento dos dados segundo as etapas da técnica CMP, comparando-se os resultados obtidos para as simulações dos levantamentos sísmicos de reflexão efetuados abaixo e acima do ângulo crítico $\left(\theta_{\mathrm{c}}\right)$.

Supondo que dentro da janela de afastamentos selecionada não ocorram mudanças bruscas na fase do sinal refletido, a principal diferença entre o processamento dos sinais abaixo e acima do ângulo crítico é o resultado do processo de análise de velocidades, através dos procedimentos convencionais, como apresentado na próxima seção.

\section{Análise dos Dados}

Para gerar os sismogramas sintéticos utilizou-se o programa SEIS88 (Cervený and Pšenøik, 1988). Apenas as reflexões foram modeladas. Inicialmente, considerouse um modelo de camadas planas (Modelo 1), cuja simulação da análise de ruído é apresentada na Figura 1.

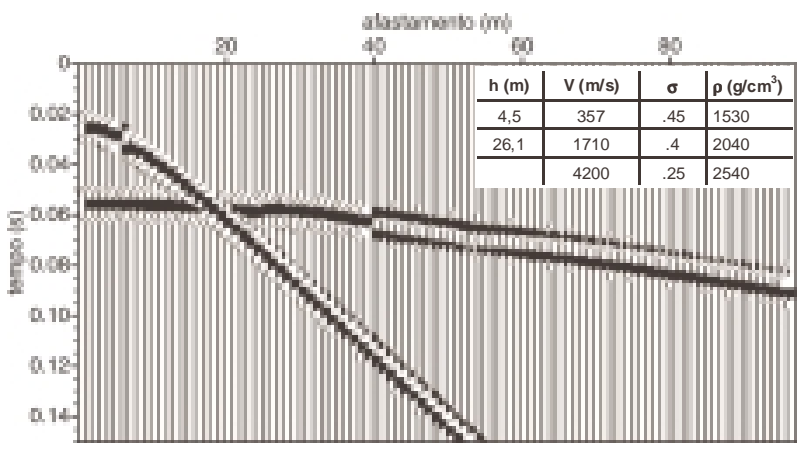

Figura 1: Sismograma sintético para os parâmetros do modelo representados no canto superior, onde: $\sigma$ é a razão de Poisson e $\rho$ é a densidade dos meios.

Para o modelo considerado, a distância crítica para a onda $P$ refratada no topo rochoso é de $24,1 \mathrm{~m}$. Observase na Figura 1 que a partir do afastamento $25 \mathrm{~m}$, o pulso refletido começa a apresentar uma mudança de fase. Próximo da segunda distância crítica ocorre uma mudança brusca na fase. Apenas a partir do afastamento $60 \mathrm{~m}$, a fase do sinal apresenta-se mais uniforme para todos os traços, o que pode ser melhor observado após a correção de NMO (Figura 2).

A janela de afastamentos entre 25 e $40 \mathrm{~m}$ também não apresenta variações bruscas na fase, entretanto a partir de $25 \mathrm{~m}$ ocorre a chegada do sinal refratado (não ilustrado na Figura 1), de modo que nos afastamentos em seguida à distância crítica para $a$ onda $P$ refratada pode haver uma distorção no pulso refletido devido à superposição de parte do pulso refratado.

Acima de $60 \mathrm{~m}$ é possível utilizar uma janela de afastamentos maior para a aquisição do levantamento de reflexão, diminuindo consideravelmente o tempo de execução do trabalho de campo. Assim, propõe-se para a situação apresentada acima investigar o uso do afastamento mínimo igual a $60 \mathrm{~m}$ para a execução do levantamento de reflexão, segundo a técnica CMP. 
Para um modelo de camadas planas, a avaliação do processo de análise de velocidades pode ser efetuada sobre o próprio sismograma da análise de ruído, como ilustrado na Figura 2. A velocidade que corrige o NMO (normal moveout) para a curva de tempo de percurso abaixo de $\theta_{\mathrm{c}}(\mathrm{VNMO}=1290 \mathrm{~m} / \mathrm{s})$ equivale à velocidade de rms do modelo (Figura 1) utilizado para gerar os dados sintéticos. Já a velocidade de NMO para as reflexões acima de $\theta_{\mathrm{c}}$ é superestimada $\left(\mathrm{VNMO}_{\mathrm{NMO}}=1450 \mathrm{~m} / \mathrm{s}\right)$. Observe na Figura 2 (marcado em vermelho), a equivalência entre as fases dos sinais abaixo e acima de $\theta_{c}$, em que deve ser lido o tempo normal. A forma de onda utilizada para gerar o sismograma sintético é uma wavelet de fase zero, por isso o tempo normal é lido na posição da amplitude máxima do sinal situado dentro da janela abaixo de $\theta_{\mathrm{c}}$.

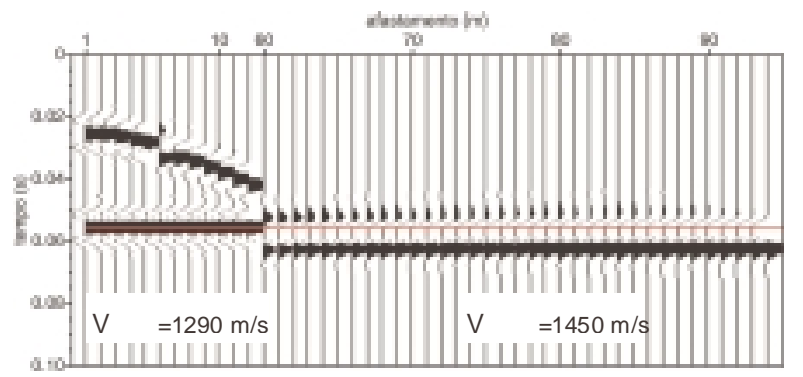

Figura 2: Sismograma apresentado na Figura 1, corrigido de $\mathrm{NMO}$, para a janela selecionada abaixo do ângulo crítico (entre 1 e $12 \mathrm{~m}$ ) e acima do ângulo crítico (a partir de $60 \mathrm{~m})$.

Na Figura 3 é apresentado o resultado do empilhamento para o sinal refletido abaixo de $\theta_{\mathrm{c}}$ utilizando a geometria CMP convencional (L1), e para o sinal refletido acima de $\theta_{c}$, empregando os levantamentos CMP convencional (L2) e o denominado por base-fixa (L3). Na Tabela abaixo são mencionados os parâmetros da geometria de aquisição de cada levantamento. Para as três simulações foram efetuados 24 pontos de tiro. A geometria base-fixa oferece a vantagem do espaço amostrado em subsuperfície, para a mesma quantidade de pontos de tiro, ser maior do que para a geometria convencional.

\begin{tabular}{|c|l|c|c|}
\hline & \multicolumn{1}{|c|}{ Geometria } & $\begin{array}{c}\text { Janela de } \\
\text { afastamentos }(\mathrm{m})\end{array}$ & $\mathrm{dx}(\mathrm{m})$ \\
\hline L1 & Convencional & $0,5-12$ & 0,5 \\
\hline L2 & Convencional & $60-83$ & 1 \\
\hline L3 & base-fixa & $60-94$ & 1 \\
\hline
\end{tabular}

Tabela1: Parâmetros da geometria de aquisição CMP, onde $d x$ é o intervalo entre receptores e entre pontos de tiro.

A Diferença entre as seções abaixo e acima de $\theta_{c}$ está na fase do pulso, ou seja, no instante de tempo, em que o refletor deve ser identificado, conforme indicado pela linha vermelha na Figura 3. Observa-se, portanto, que a imagem estrutural do refletor em subsuperfície obtida para os levantamentos abaixo e acima de $\theta c$ são equivalentes. O problema surge na etapa da conversão da seção em tempo para profundidade, o que requer os valores do modelo de velocidades intervalares da onda $P$.

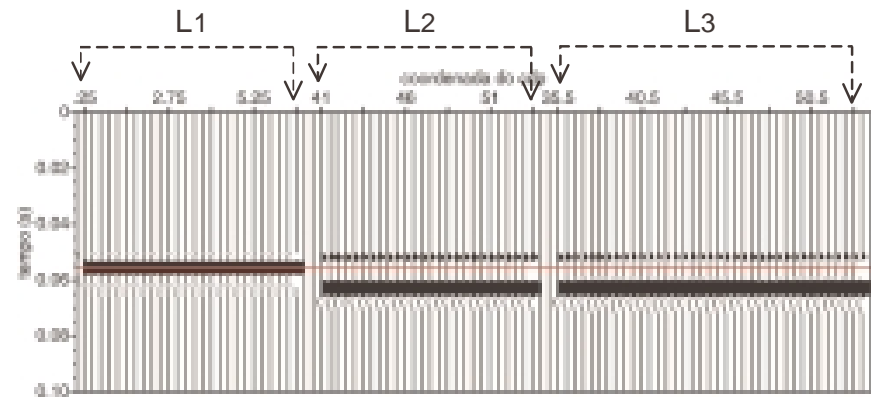

Figura 3: Seções empilhadas obtidas para geometrias de aquisição diferentes (ver descrição no texto). A escala horizontal marca a coordenada do ponto CMP considerando que o primeiro ponto de tiro está na coordenada zero $(\mathrm{m})$.

Como a Vnmo encontrada acima do ângulo crítico não equivale à Vrms, não é possível utilizar a fórmula de Dix (1955) para o cálculo das velocidades intervalares. Neste caso, uma boa estimativa para as velocidades intervalares pode ser obtida através da interpretação das refrações observadas nos próprios sismogramas de campo obtidos para o levantamento CMP, e programando-se a aquisição de alguns sismogramas adicionais concomitantes à aquisição CMP para a obtenção do tiro reverso e da velocidade da onda direta.

Para ilustrar a influência dos parâmetros de elasticidade na forma do pulso refletido foi gerado um sismograma sintético para o modelo apresentado na Figura1, modificando o valor da Razão de Poisson da camada 3 para $(\sigma 3=.3)$ (Modelo 2), simulando um embasamento fraturado, por exemplo. A Figura 4 refere-se ao sismograma da análise de ruído e a Figura 5 às seções empilhadas para as geometrias L1, L2 e L3 (Tabela 1).

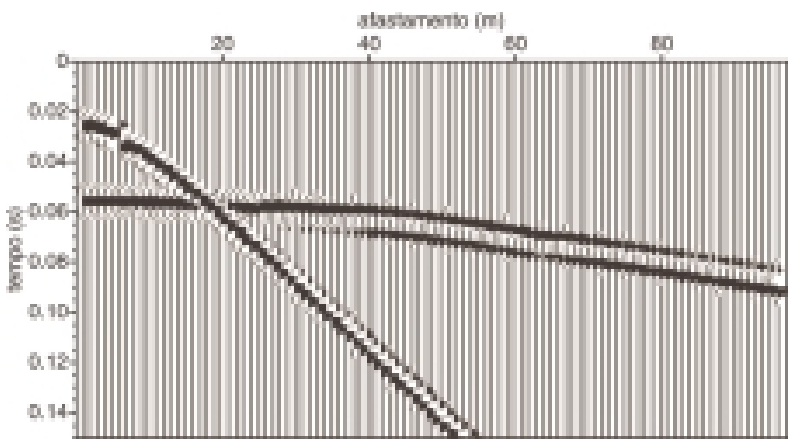

Figura 4: Sismograma sintético para os parâmetros do modelo representados no canto superior da Figura 1, substitu indo o valor da Razão de Poisson para a camada 3 para $(\sigma 3=.3)$.

O mesmo fator de ganho foi empregado em todas as seções das Figuras 3 e 5. Nota-se que as imagens da estrutura do refletor são equivalentes dentro da janela de afastamentos selecionada. A diferença entre a defasagem dos sinais refletidos no topo rochoso depois do empilhamento não é tão evidente quanto nos sismogramas para análise de ruído (Figuras 1 e 4). 


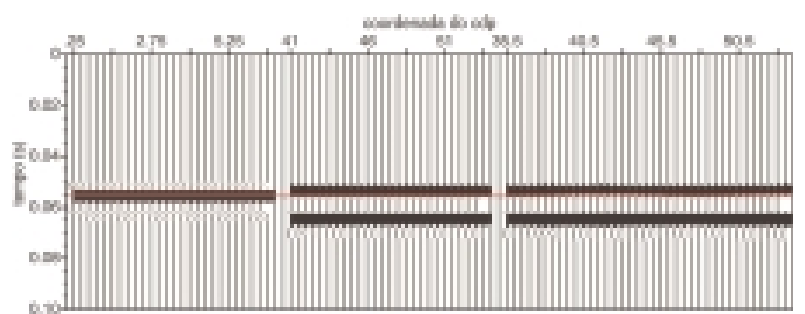

Figura 5: Seções empilhadas, para o modelo utilizado para gerar o sismograma (Fig. 4), empregando as mesmas geometrias das seções da Fig. 3.

As distâncias críticas mudam com a profundidade, e quando a interface não for aproximadamente plana, a janela selecionada com base na análise de ruído pode não se manter ideal para toda a linha. Para ilustrar, considerou-se um refletor inclinado (Figura 6) e geraramse dois sismogramas para análise de ruído com os pontos de tiro fixos nas coordenadas $90 \mathrm{~m}$ e $120 \mathrm{~m}$, para os mesmos valores de velocidade, razão de Poisson e densidade do Modelo 1 (Figura 7) e do Modelo 2 (Figura 8).

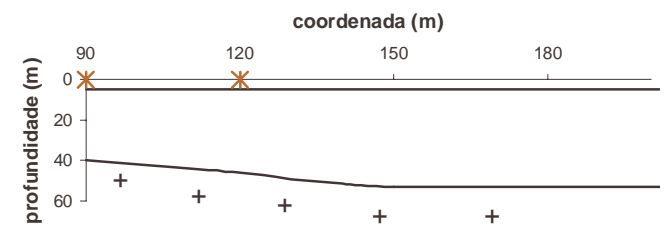

Figura 6: Interfaces dos Modelos 3 e 4.

(a)
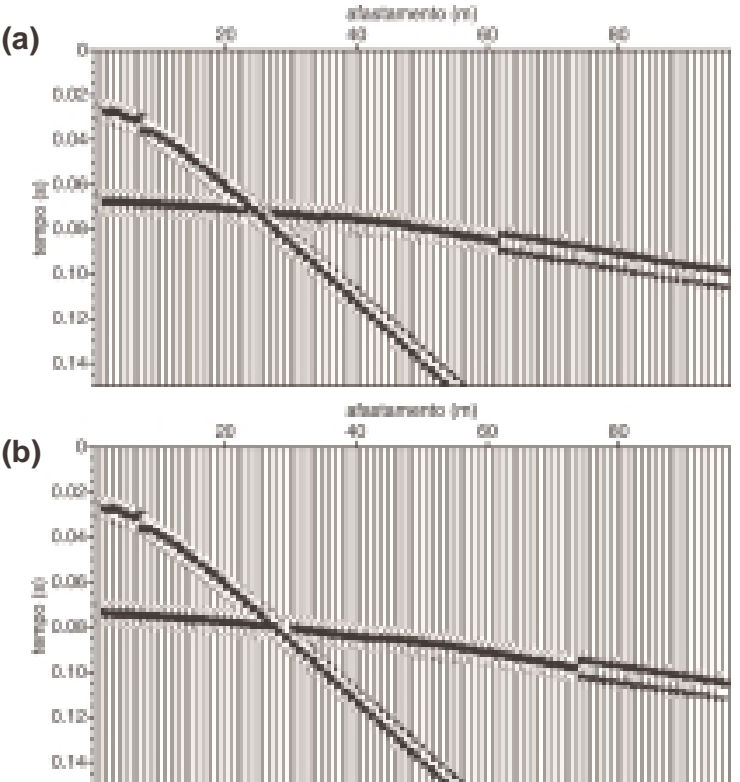

Figura 7:Sismogramas gerados para o Modelo 3 (Figura 6, com valores de V, $\sigma$ e $\rho$ do Modelo 1 (Fig. 1)): pt1 (a) e pt2 (b), respectivamente nas coordenadas $90 \mathrm{~m}$ e $120 \mathrm{~m}$.
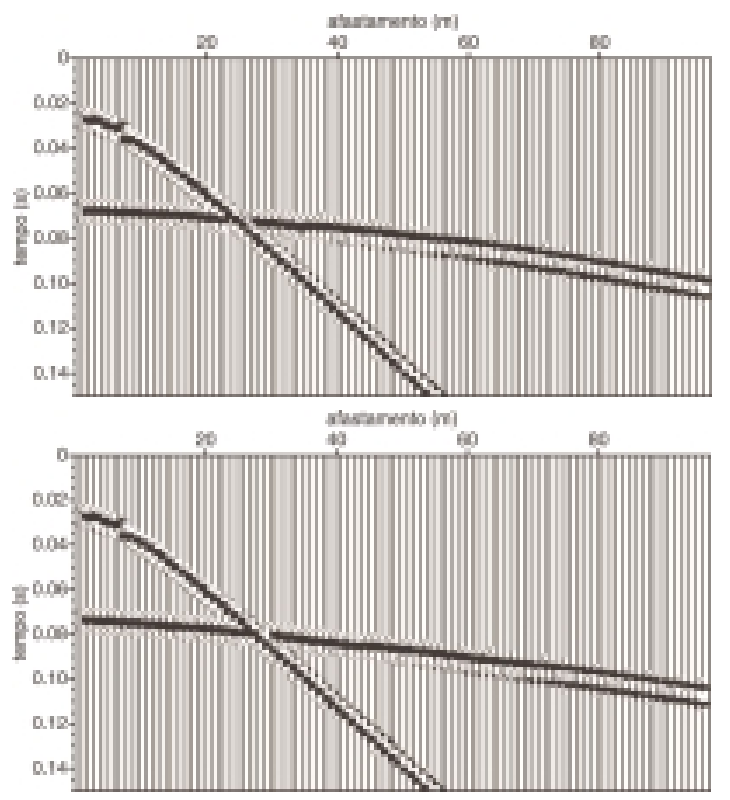

Figura 8: Sismogramas gerados para o Modelo 4 (Figura 6, com valores de V, $\sigma$ e $\rho$ do Modelo 2 (Fig. 4)): pt1 (a) e pt2 (b), respectivamente nas coordenadas $90 \mathrm{~m}$ e $120 \mathrm{~m}$.

Para o refletor inclinado, a avaliação da equivalência entre as fases dos sinais abaixo e acima de $\theta_{c}$, na qual o refletor será identificado, deve ser efetuada sobre os sismogramas CMP, como ilustrado na Figura 9, para o Modelo 4 (Fig. 8) adotando-se como afastamento mínimo $62 \mathrm{~m}$.

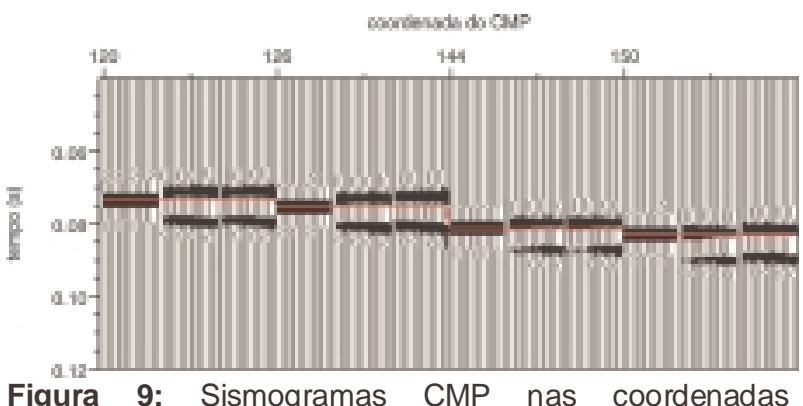
indicadas, respectivamente, para as geometrias L1, L2 e L3 (Tabela 1, porém, com afastamentos $4-15,5 \mathrm{~m}$ para L1, 62-85m para L2 e 62-96m para L3).

Na Figura 10 são apresentadas as seções empilhadas, obtidas respectivamente, para as geometrias de aquisição L1, L2 e L3 (Tabela 1, porém, com afastamentos $4-15,5 \mathrm{~m}$ para $\mathrm{L} 1,62-85 \mathrm{~m}$ para $\mathrm{L} 2$ e 62 $96 \mathrm{~m}$ para L3). A posição dos pontos de tiro não é a mesma para os três levantamentos. Os tiros foram posicionados de forma que a coordenada do primeiro CMP com multiplicidade máxima (1200\%) fosse a mesma para as três simulações. Observa-se, também, que para os levantamentos L1 e L2 foram efetuados 42 pontos de tiro, enquanto que para o L3 foram efetuados 36 , para se amostrar o mesmo espaço em subsuperfície. 


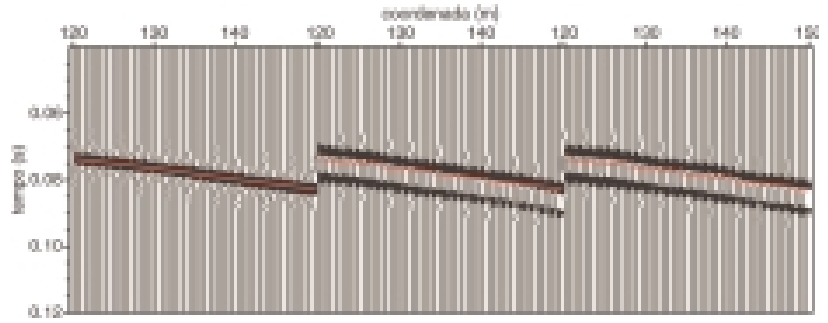

Figura 10: Seções empilhadas obtidas para diferentes geometrias de aquisição (ver descrição no texto). Em vermelho está assinalada a fase em que o refletor é identificado em cada uma das seções.

A seguir, é apresentado um sismograma para análise de ruído (Figura 11) coletado no campus da capital da Universidade de São Paulo, no terreno em frente ao prédio do Instituto de Física. Foi utilizado um sismógrafo de 24 canais (OYO Geospace), geofones com freqüência natural de $100 \mathrm{~Hz}$ e como fonte de energia, uma marreta sobre placa de metal. $O$ intervalo de amostragem em tempo foi de $0.125 \mathrm{~ms}$.

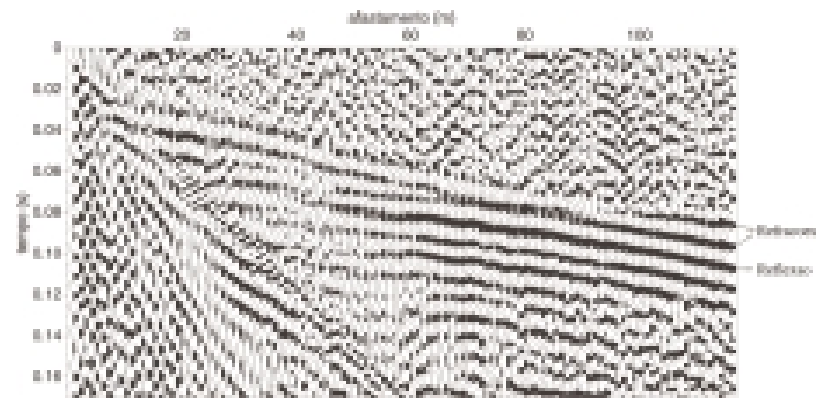

Figura 11: Sismograma para análise de ruído.

A interpretação das refrações observadas nesse sismograma, assumindo um modelo de camadas planas, forneceu o modelo velocidade-profundidade utilizado para gerar os dados sintéticos apresentados na Figura 1. Observe a concordância entre os dados sintéticos e reais para a curva de reflexão. O uso de sismogramas sintéticos gerados a partir de modelos preliminares da área investigada é uma ferramenta importante para a identificação das reflexões nos dados reais.

\section{Discussões e Conclusões}

Do estudo apresentado concluí-se que é possível obter uma imagem da estrutura correta dos refletores, através da técnica CMP para reflexões registradas acima do ângulo crítico, desde que sejam incorporadas informações adicionais sobre o modelo de velocidades da onda $\mathrm{P}$.

A aquisição acima do ângulo crítico permite o uso de uma janela de afastamentos mais ampla, tornando a execução da linha sísmica mais rápida, ao se utilizar um intervalo entre receptores maior. Além disso, tem-se mais liberdade para optar pelo uso da geometria denominada aqui, por base-fixa. Apesar de atualmente existirem sismógrafos portáteis com 96 canais, a possibilidade de trabalhar com um número menor de canais economiza tempo no campo: em plantar os geofones, lidar com os cabos e, etc.. Outra vantagem da técnica mantendo a base de geofones fixa, mesmo utilizando-se um sismógrafo com 96 canais, por exemplo, seria a possibilidade de se executar duas ou mais linhas paralelas para o mesmo ponto de tiro, similar à aquisição 3D.

O inconveniente da geometria CMP base-fixa, ao contrario da geometria convencional, é que as famílias CMP não são todas amostradas para o mesmo grupo de afastamentos. Entretanto, desde que haja uma certa uniformidade da fase do pulso refletido dentro da janela de afastamentos empregada, não ocorre uma diferença significativa para o empilhamento dos sinais amostrados em grupos de afastamentos diferentes, para a escala de investigação rasa.

Para a modelagem das reflexões apenas um aspecto da propagação de ondas foi considerado, a partição de energia na interface. Embora esse seja 0 fator predominante é necessário verificar se outros algoritmos que modelam o campo total de ondas forneceriam ajustes mais precisos aos dados observados.

O estudo realizado até o momento comprovou o potencial para a estimativa da densidade e dos módulos de elasticidade do meio explorando a defasagem do pulso refletido acima do ângulo crítico. Mas, ainda é preciso investigar qual é o grau de ambigüidade do problema em questão e concluir a implementação de um procedimento automático para a busca do modelo que melhor ajusta os dados modelados aos observados.

\section{Agradecimentos}

Ao Dr. Renato L. Prado e ao Dr. Marcelo S. de Assumpção pelas proveitosas discussões.

\section{Referências}

Cervený, V., Pšenøík, I., 1988. Program package SEIS88.

Dix, C.H., 1955. Seismic velocities from surface measurements. Geophysics, 20: 68-86.

Hunter, J. A., Burns, R. A. \& Good, R. L., 1980. Optimum field techniques for bedrock reflection mapping with the multichannel engineering seismograph: Presented at the $50^{\text {th }}$ Ann. Internat. Mtg. and Expos., Soc. Of Explor. Geophys., Houston

Le Diagon, F. M. M., 2000. Investigações sobre metodologias de aquisição e interpretação de dados sísmicos de reflexão rasa para imageamento do topo rochoso. Dissertação de Mestrado, Departamento de Geofísica da Universidade de São Paulo, Brasil.

Pullan, S. E. \& Hunter, J. A., 1985. Seismic model studies of the overburden-bedrock reflection. Geophysics, 50: 1684-1688. 